\title{
El rol de los parlamentos en garantizar el acceso universal a internet como derecho humano ${ }^{1}$
}

\section{The Role of Parliaments in Guaranteeing Universal Access to Internet as a Human Right}

Gabriela A. Commatteo

Resumen: Las transformaciones que ha vivido la humanidad en las últimas décadas, desde la invención de la computadora, Internet y -más recientemente- la robótica, la impresión 3D, el Big Data, la Inteligencia Artificial, entre otros, suscitan la imperiosa necesidad de brindar un marco de protección que avance sobre una nueva "generación” de derechos. Una “cuarta generación” debería incluir, entre otros, el derecho a la información, a un Estado abierto, a una democracia plena, a la privacidad en la era digital, a la igualdad e inclusión en el ámbito digital. En el pasado, a medida que se desarrollaban los marcos de protección para las diferentes "generaciones" de derechos humanos, los parlamentos cumplieron un rol fundamental receptando esos derechos en sus ordenamientos internos. En ese contexto, este trabajo explorará las características de Internet como derecho humano, los desafíos que presenta la brecha digital que impiden expandir el acceso universal a Internet y el rol

\footnotetext{
${ }^{1}$ Este trabajo de investigación fue llevado a cabo en el marco del Proyecto IUS 08002019 0100017 CT, "Los derechos fundamentales ante los desafíos de la era digital: Una búsqueda de soluciones para Argentina en un contexto global", de la Facultad de Derecho de la Universidad Católica Argentina.

2 Coordinadora del Proyecto IUS. Abogada y Traductora Pública en Inglés por la Universidad Católica Argentina. Becaria Chevening, cursando Master of Law (LLM) in Information Technology and Intellectual Property en King's College London.
} 
fundamental que cumplen los parlamentarios en poder garantizarlo, en un contexto global y, en particular, en el ámbito de la Argentina. Asimismo, analizará cómo la cooperación internacional, a través de la diplomacia parlamentaria, sirve como una herramienta para cooperar en la garantía del acceso a Internet y en la gobernanza de Internet, a fin de promover sociedades más justas, igualitarias y democráticas.

Palabras clave: Derechos digitales - Acceso a Internet Parlamento - Diplomacia parlamentaria

Abstract: The transformations that humanity has undergone in recent decades, since the invention of the computer, the Internet and -more recently- robotics, 3D printing, Big Data, Artificial Intelligence, among others, raise the urgent need to provide a protection framework that advances a new "generation" of rights. A "fourth generation" of rights must include, among others, the right to information, to an Open State, to a full democracy, to privacy in the digital era, to digital equality and inclusion. In the past, as the protection frameworks for the different "generations" of human rights were developed, parliaments played a fundamental role by incorporating those rights to domestic legal systems. In this context, this paper will explore the features of Internet as a human right, the challenges posed by the digital divide that prevent the expansion of universal access to Internet, and the fundamental role that parliamentarians play in guaranteeing it, both in the global context and specifically within the Argentine Republic. Likewise, it will analyze how international cooperation in parliamentary matters through the so-called parliamentary diplomacy serves as a tool for legislators to cooperate in guaranteeing Internet access and Internet governance in order to promote more just and egalitarian societies.

Key words: Digital Rights - Access to Internet - Parliament Parliamentary Diplomacy 
Recibido: 17.3.2021Ａceptado: 15.6.2021

Sumario

\section{Introducción}

2. Internet como derecho humano

3. La brecha digital y los objetivos de desarrollo sostenible

4. El rol de los parlamentarios en garantizar el acceso a Internet

5. El rol de los parlamentos en el ámbito internacional

\section{Conclusiones}

\section{Introducción}

Los derechos humanos son reconocidos como los principales deberes de los Estados para con sus gobernados. En un primer estadio se buscó proteger derechos fundamentales como la vida y la libertad. Documentos constitucionales fundacionales para Inglaterra como la Carta Magna de 1215 y el Bill of Rights de 1628, más tarde la Declaración de los Derechos del Hombre y del Ciudadano de 1789 en Francia y el Bill of Rights norteamericano de 1791 consagraron derechos cardinales, conocidos como derechos de primera generación, que luego se reflejaron en la Declaración Universal de los Derechos Humanos de 1948.

Más tarde, la segunda ola de protección contempló otros derechos: los económicos, sociales y culturales. Se avanzó así a proteger, por ejemplo, el derecho al trabajo, la libertad sindical, el derecho a la salud o la educación. Constituciones nacionales y diversos pactos y tratados reflejaron su protección en el siglo XIX y, fundamentalmente, durante todo el siglo XX.

La tercera generación fue un paso más allá, promoviendo, durante las últimas décadas del siglo $\mathrm{XX}$, la protección de los 
"derechos del pueblo o la solidaridad". Derechos que instan a la cooperación entre las naciones, entre diversos grupos y organismos, y que se enfocan particularmente en la paz, el derecho al desarrollo y la protección del medio ambiente para el disfrute de todos.

La división en generaciones se debe a Karel Vasak ${ }^{3}$, asesor de la UNESCO, quien desarrolló esta teoría en un artículo (Vasak, 1977). Es importante destacar que existen posturas que critican la metáfora de las "generaciones" de Vasak, como es el caso de Macklem, quien afirma que "la concepción generacional pasa por alto el hecho de que, a pesar de los diversos conjuntos de intereses que se protegen, los derechos humanos en el derecho internacional comparten un propósito común (...) comprenden solo una generación: una sola población de derechos" (Macklem, 2015, p. 62).

Sin embargo, considero que la metáfora de la división generacional de Vasak es útil, ya que enfatiza hechos cronológicos importantes acerca de los derechos humanos. De hecho, las transformaciones que ha vivido la humanidad en las últimas décadas, desde la invención de la computadora, Internet y -más recientemente- la robótica, la impresión 3D, el Big Data, la Inteligencia Artificial, entre otros, suscitan la imperiosa necesidad de brindar un marco de protección que avance sobre una nueva "generación" de derechos: un marco innovador. ¿Cómo innovamos en la protección de los derechos humanos? ¿Qué derechos humanos son "innovadores" en medio de la llamada "cuarta" ${ }^{4}$ revolución industrial?

Creo que la "cuarta generación" de derechos incluye, entre otros, el derecho a la información, a un Estado abierto, a una democracia

\footnotetext{
${ }^{3}$ En noviembre de 1977, Karel Vasak, asesor legal de la UNESCO y distinguido experto en derechos humanos, escribió un artículo para el UNESCO Courier, donde presentaba la idea de las tres generaciones de derechos humanos. https://www.openglobalrights.org/putting-to-rest-the-three-generations-theory-ofhuman-rights/?lang=Spanish

${ }^{4}$ No existe una única corriente que determine la cantidad de revoluciones industriales que se han suscitado hasta el momento, pero, en términos generales, la mayoría de los autores se refiere a la actual como la cuarta revolución industrial.
} 
plena, a la imagen digital, a la privacidad en la era digital, a la igualdad e inclusión. Llamativamente, en el siglo XXI, no podemos hablar de desarrollo sostenible ni de inclusión sin pensar en la democratización de la digitalización, del acceso a la Tecnología de la Información y las Comunicaciones (TIC). Muy pocos desarrollos tecnológicos han tenido el efecto revolucionario que ha producido Internet.

Mientras la pandemia generada por el COVID-19 transforma la realidad laboral, la manera en la que nos relacionamos, estudiamos y adquirimos bienes esenciales, nunca antes ha sido tan importante reducir la brecha digital para los 3.600 millones de personas que aún no tienen conexión a Internet y no pueden acceder a la educación en línea, al empleo o a consejos de salud y saneamiento claves.

A medida que se desarrollaban los marcos de protección para las diferentes "generaciones" de derechos humanos, los parlamentos tuvieron un rol fundamental receptando esos derechos en sus ordenamientos internos. Asimismo, fueron innovadores en el ámbito de la cooperación internacional. Hace 131 años - mucho antes de la Primera Guerra Mundial, de la invención de las vacunas, de la creación de Internet, de la digitalización, incluso mucho antes del surgimiento de la ONU-,con la creación de la Unión Interparlamentaria nacía la idea de una diplomacia parlamentaria organizada. Considero que la diplomacia parlamentaria es una herramienta fundamental al servicio de los parlamentarios que, hoy en día, les permite avanzar en la protección de derechos fundamentales como el acceso a Internet.

En este trabajo se explorarán las características de Internet como derecho humano, los desafíos que presenta la brecha digital que impiden expandir el acceso universal y el rol fundamental que cumplen los parlamentarios en poder garantizarlo. La problemática se abordará desde el punto de vista global, dado que este tipo de tecnologías traspasan todas las fronteras y sistemas jurídicos nacionales. Sin embargo, se pondrá especial foco sobre la perspectiva argentina. 
Asimismo, se analizará cómo la cooperación internacional en materia parlamentaria, a través de la llamada diplomacia parlamentaria, sirve como una herramienta al servicio de los legisladores para cooperar en la garantía del acceso a Internet y en la gobernanza de Internet, a fin de promover sociedades más justas, igualitarias $\mathrm{y}$ democráticas $\mathrm{y}$ garantizar otros derechos fundamentales.

\section{Internet como derecho humano}

\section{a. ¿Es Internet en sí mismo un derecho?}

En 2010, el BBC World Service llevó adelante una encuesta en 26 países, en la cual se entrevistó a más de 27.000 adultos. Cuatro de cada cinco $(79 \%)$ consideró que Internet era un derecho fundamental que les correspondía. El $87 \%$ de todos los entrevistados que utilizaban Internet declaró que el acceso a Internet debería ser "un derecho fundamental de la humanidad" (BBC, 2010).

En 2011, variados medios de comunicación se apresuraron a dar la gran noticia: finalmente las "Naciones Unidas declaran el acceso a Internet como derecho humano ${ }^{5 ”}$. Sin embargo, no fue una declaración de derecho humano per se lo que sucedió en ese momento.

En realidad, el hito que tanto resonó en los medios de comunicación fue la presentación de un informe por parte del Relator Especial sobre la Promoción y Protección del Derecho a la Libertad de Opinión y de Expresión, Frank La Rue, ante el Consejo de Derechos Humanos de las Naciones Unidas. En el informe se estudian las principales tendencias y desafíos al derecho de todas las personas a

\footnotetext{
5 Ver entre otros: Hipertextual https://hipertextual.com/2011/06/la-onu-declara-elacceso-a-internet-como-derecho-humano; CNN https://cnnespanol.cnn.com/2011/06/09/el-acceso-a-internet-un-derecho-humanosegun-la-onu/ CLAD http://old.clad.org/noticias/la-onu-declara-el-acceso-ainternet-como-un-derecho-humano-fundamental-2
} 
buscar, recibir y difundir información e ideas de todo tipo por Internet (Organización de las Naciones Unidas [ONU], 2011). Asimismo, entiende que Internet ha pasado a ser un instrumento indispensable para ejercer diversos derechos. La Rue explica que "la meta del acceso universal a Internet ha de ser prioritaria para todos los Estados" (ONU, 2011, p.24). Esto no implica una declaración de Internet como derecho humano, aunque demuestra un gran avance.

En este sentido, es importante destacar que el Relator Especial considera que "Internet es uno de los instrumentos más poderosos del siglo XXI para exigir más transparencia en la conducta a quienes ejercen el poder, acceder a información y facilitar la participación ciudadana activa en la forja de sociedades democráticas" (ONU, 2011, p.4). Agrega que le preocupa que sin ese acceso que "facilita el desarrollo económico y el disfrute de diversos derechos humanos, los grupos marginados y los Estados en desarrollo sigan atrapados en su situación de desventaja, que perpetúa la desigualdad entre Estados y dentro de un mismo Estado" (ONU, 2011, p. 18).

En julio de 2016, la Asamblea General de las Naciones Unidas adoptó una declaración no vinculante sobre la "Promoción, protección y disfrute de los derechos humanos en Internet”, en la cual se destaca "la importancia de que se aplique un enfoque basado en los derechos humanos para facilitar y ampliar el acceso a Internet, y que Internet sea abierta, accesible y cuente con la participación de múltiples interesados" (ONU, 2016, p. 3). De esta forma, mediante tal declaración (A/HRC/32/L.20) se dio un paso más en el convencimiento de que el acceso a Internet debe entenderse como un derecho humano, que a la vez se presenta como catalizador de otros derechos. Sin embargo, no contó con el apoyo de países como Rusia, China o Sudáfrica.

En 2019, la Universidad de Birmingham publicó una investigación realizada por el Dr. Merten Reglitz, profesor de ética global de la mencionada universidad, en el que se afirma que el acceso a Internet debería ser considerado un derecho humano. En su 
análisis, Reglitz asevera que "Internet es necesario (o al menos tiene una importancia crucial) para la concreción de derechos humanos y el fomento de la democracia (...)" y, en los mismos términos ya expresados por la ONU en los mencionados informes, entiende que "expandir las capacidades de los individuos (...) contribuye al progreso de la humanidad en su conjunto” (Reglitz, 2019, p.1). Asimismo, concibe que, en un mundo cada vez más globalizado y virtual, Internet es esencial para el ejercicio de ciertos derechos humanos políticos, como la libertad de expresión, asociación, entre otros, mientras que su gobernanza adecuada permitiría proteger otros derechos humanos fundamentales (la vida, la libertad, etc.) (Reglitz, 2019).

\section{b. El derecho de Acceso a Internet y la Constitución Nacional}

Las constituciones nacionales son instrumentos jurídicos con vocación para proteger, a largo plazo, los derechos fundamentales de los ciudadanos. Afirma Bidegain que "la constitución consiste en la ordenación fundamental del Estado” (Bidegain, 1994; 2001, p. 11), que debe poner en práctica las políticas públicas y leyes necesarias para garantizar el cumplimiento de esos derechos. Conforme al concepto racional-normativo, por lo general, los procesos de reforma de las constituciones prevén requisitos mucho más difíciles de cumplir que cualquier otra ley (Bidegain, 1994; 2001). Ello nos hace inferir que los constituyentes deben adelantarse a los cambios que vendrán y lograr que el texto perdure en el tiempo. De esta forma, la Constitución Nacional ha sufrido muy pocos cambios desde 1853-1860, siendo el último —en 1994- el más trascendental de todos.

Aun así, con la incorporación de los llamados derechos de tercera generación en la reforma, no era posible que nuestros constituyentes previeran la ola de innovación que inundaría el mundo durante los siguientes veinticinco años. Un proceso de avance y desarrollo que ya había comenzado antes de la citada reforma, pero que sin dudas se aceleró profundamente con los adelantos tecnológicos, las 
tecnologías disruptivas, y que se precipitó muchísimo más por la pandemia del COVID-19. Era impensado, en ese momento, consagrar en la Constitución Nacional el reconocimiento de los derechos de cuarta generación; era impensado incluir garantías de acceso a Internet y a las TIC.

En principio, no sería un problema que Internet no esté reconocido per se como un derecho en nuestra constitución para garantizar su acceso. De hecho, nuestra constitución establece, en el artículo 33, conocido como el artículo sobre "derechos implícitos", que "las declaraciones, derechos y garantías que enumera la Constitución no serán entendidos como negación de otros derechos y garantías no enumerados; pero que nacen del principio de la soberanía del pueblo y de la forma republicana de gobierno".

Sumado a ello, Rothkopf expresa que "afirmar que las personas no pueden reivindicar derechos que superen la imaginación de los constitucionalistas es una ridiculez, y una ridiculez de por cierto peligrosa” (Rothkopf, 2015).

Sin embargo, y más allá del reconocimiento implícito o la falta de inclusión del derecho, creo que nuestros constitucionalistas fueron grandes visionarios al incluir, con espíritu previsor, una cláusula fundamental para nuestro desarrollo futuro. Así el nuevo artículo 75, inciso 19, viene a ampliar la llamada "cláusula del progreso" del inciso 18. De esta forma se establece que corresponde al Congreso:

Proveer lo conducente al desarrollo humano, al progreso económico con justicia social, a la productividad de la economía nacional, a la generación de empleo, a la formación profesional de los trabajadores, a la defensa del valor de la moneda, a la investigación $y$ al desarrollo científico y tecnológico, su difusión y aprovechamiento. Proveer al crecimiento armónico de la Nación y al poblamiento de su territorio; promover políticas diferenciadas que tiendan a equilibrar el desigual desarrollo relativo de provincias y 
regiones. Para estas iniciativas, el Senado será Cámara de origen. (Constitución de la Nación Argentina).

Entiendo que este artículo debería interpretarse a la luz del contexto actual del avance de la digitalización, para lo cual el acceso a Internet se presenta a la vez como una herramienta y un derecho fundamental.

Así lo expresaba Jennifer Ferguson-Mitchell, representante de la Unión Internacional de Telecomunicaciones (UIT) al sostener que "las tecnologías de la información y la comunicación están cambiando la respuesta del mundo a los desafíos que enfrenta la humanidad. Estas ayudan a los esfuerzos humanitarios para llegar a más personas y mejorar la calidad de vida de millones de comunidades vulnerables en nuestro planeta" (ONU News, 2017).

Es decir que el "desarrollo humano" al que se refiere el inciso en la manda constitucional, no puede alcanzarse en forma plena sin contemplar la garantía del acceso a Internet. Entiendo que, incluso aunque no haya sido previsto de esta forma, el "desarrollo tecnológico, su difusión y aprovechamiento” debería interpretarse como el desarrollo necesario para que Internet pueda llegar a todos los habitantes del país. Internet ofrece oportunidades sin precedentes para la realización de los derechos humanos y desempeña un papel cada vez más importante en nuestra vida cotidiana:

El hombre de la informatización se beneficia, tanto como las instituciones, del avance tecnológico, y por primera vez contamos con vías de acceso a la información que con una inversión mínima permiten un alcance máximo. Esto no supone de manera automática un elemento democratizador de los medios de producción, pero no cabe duda de que es una dinámica que cambia la orientación centralista que había tenido la tecnología, antes de la era computarizada (Aguila, 2012).

El derecho de acceso a Internet, que también se conoce como el derecho a la banda ancha o a conectarse, no solo implica que 
mediante la conexión se puede lograr la concreción del acceso a la información, sino que involucra la realización de muchos otros derechos. Para esto es fundamental trabajar en el cierre de la brecha digital. A continuación, se explica la importancia de cerrar la brecha digital, dado que al universalizar el acceso a Internet estaríamos promoviendo el desarrollo y progreso de la sociedad en su conjunto, ya que este cumple un rol fundamental en la concreción de los Objetivos de Desarrollo Sostenible de la Agenda 2030, adoptada por la Asamblea General de Naciones Unidas, como se expondrá a continuación.

\section{La brecha digital y los objetivos de desarrollo sostenible}

A la luz del análisis desplegado hasta aquí, no es absurdo afirmar, hoy en día, en el año 2021, que Internet es un derecho humano. Además, como se ha mencionado en párrafos anteriores, Internet es, sin lugar a dudas, una herramienta fundamental para la promoción y realización de otros derechos.

Dentro del marco de protección internacional, los informes de la ONU, así como de otros organismos internacionales como la Organización de los Estados Americanos (OEA) o la Comisión Interamericana de Derechos Humanos (CIDH), establecen que no debería imponerse ninguna restricción sobre el acceso a Internet, ya que se estarían violando derechos tan importantes como la libertad de expresión. Es decir, solo podrían bloquearse ciertos sitios web, como una acción extrema y ante delitos muy graves.

En el marco interno argentino, el reconocimiento como derecho humano se podría inferir de diferentes artículos de la Constitución, como el mencionado artículo 33, aunque también se ha promovido a través de leyes tales como la Ley Argentina Digital N²7.078, que, si bien no lo declara derecho humano, lo reconoce como una tecnología preponderante para la concreción del derecho humano a las 
comunicaciones. Sin embargo, esta mención resulta insuficiente a la luz de la importancia que este derecho ha cobrado en las últimas décadas.

Más allá de los beneficios que ha traído para el hombre de "la era de la información”, en relación con la libertad de expresión y el acceso a la información, Internet tiene también una enorme influencia en otros derechos: permite acceder al derecho a la educación, que repercute sin dudas en el desarrollo humano; conjuntamente, brinda acceso a la cultura, facilita la comunicación, amplia el derecho de reunión y asociación en el marco de la globalización. Como si esto no fuera suficiente motivo para garantizar su acceso, la pandemia producida a raíz del brote de COVID-19 ha evidenciado fundamentalmente como Internet cumple un rol protagónico en garantizar el acceso a la salud, el trabajo y tantos otros derechos.

El Relator Especial afirma en el precitado informe de 2011 que:

Aunque Internet existe desde 1960, su actual uso en el mundo entero por distintos grupos de edad y su incorporación en prácticamente todos los aspectos de la vida humana moderna no tiene precedentes. Según la UIT, el número total de usuarios de Internet en todo el mundo supera actualmente los 2.000 millones de personas. El número de usuarios activos de Facebook pasó de 150 millones a 600 millones entre 2009 y 2011 (ONU, 2011, p. 4).

Actualmente, esas cifras han cambiado en forma considerable. La UIT reveló, a fines de 2019, que los usuarios de Internet ascendían a 4.100 millones de personas (UIT, 2019), un aumento del $50 \%$ en tan solo 8 años. Según el informe "Digital 2020", de We Are Global, la cantidad de usuarios de Internet se incrementó, llegando a casi 4.500 millones de personas, de los cuales 3.800 millones son usuarios de redes sociales. Casi el $60 \%$ de la población mundial se encuentra online (We are Global, 2020). El informe también muestra como, en 2019, Facebook ya contaba con 2.271 millones de usuarios a nivel 
global. El aumento, en un período de tan solo 8 años, es ciertamente exponencial.

Contemplar estas cifras fuera de contexto resultaría en la conclusión errónea de que el crecimiento y el alcance de Internet no requiere de otras acciones, ya que una parte considerable de la población tiene posibilidades de acceso. Cabe, entonces, plantear el siguiente interrogante.

\section{a. ¿Por qué hablamos de brecha digital?}

A fin de proyectar mayor comprensión sobre el tema, primero debemos definir a qué nos referimos con "brecha digital". La Rue, enuncia en su informe que "con la expresión brecha digital se hace referencia a la separación entre quienes tienen acceso efectivo a las tecnologías digitales y de la información, en particular a Internet, y quienes tienen un acceso muy limitado o carecen de él" (ONU, 2011, p. 18). El $40 \%$ de la población mundial sigue desconectada. 3.600 millones de personas en el mundo aún no acceden. Más de mil millones de esos "desconectados" viven en el Sudeste Asiático. Los desconectados del continente africano representan el $27 \%$, con 870 millones de personas que todavía no pueden conectarse en todo el continente. En otros términos, el $65 \%$ de los habitantes del continente africano no poseen acceso a Internet (We Are Social, 2020). Las causas de la falta de conexión en estos territorios son diversas, y podrían referir tanto a factores económicos que hacen al desarrollo como a cuestiones políticas. Sin embargo, el análisis de esas causas no es materia de este artículo.

La Rue continúa diciendo:

Asimismo, existen brechas digitales en función de la riqueza, el género y demarcaciones geográficas y sociales dentro de un mismo Estado. De hecho, en vista de que la riqueza es uno de los principales factores que determinan quién puede acceder a las tecnologías de la información y las comunicaciones, es probable que el acceso a Internet se concentre en las élites 
socioeconómicas, especialmente en los países donde Internet no está muy extendido (ONU, 2011, p. 18).

En septiembre de 2015, se reunieron en Nueva York los representantes de 170 países del mundo, para participar de la Cumbre de la ONU sobre el Desarrollo Sostenible. Allí aprobaron -mediante la resolución A/RES/70/1 - la Agenda 2030, que comprende 17 Objetivos de Desarrollo Sostenible (ODS). A la vez, estos engloban 167 metas conexas, que servirían de marco general para orientar las actividades de desarrollo a escala nacional y mundial hasta $2030^{6}$.

El objetivo más relevante para el tema que nos convoca es el $O D S$ 9 "Industria, Innovación e Infraestructura" que establece en su Meta 9.c: "Aumentar significativamente el acceso a la tecnología de la información y las comunicaciones y esforzarse por proporcionar el acceso universal y asequible a Internet en los países menos adelantados a más tardar en 2020".

Es decir que, a pesar de que no exista una declaración formal de derecho humano, la ONU, a través de sus acciones realizadas con el Foro de Gobernanza de Internet ${ }^{7}$, en primer lugar y, por sobre todo, al incluir esta meta en un ODS, entiende que el acceso a Internet debe ser universal, porque todos debemos gozar de este derecho, catalizador de otros derechos.

Dentro de los 17 ODS, pueden identificarse diversas metas que ya tenían incorporadas las TIC:

- ODS 1 Fin de la Pobreza: "Para 2030, garantizar que todos los hombres y mujeres, en particular los pobres y los más vulnerables, tengan los mismos derechos a los recursos

\footnotetext{
6 Para más información sobre la Agenda 2030 para el Desarrollo Sostenible: https://www.un.org/sustainabledevelopment/es/development-agenda/

7 En 2003 y 2005, las Naciones Unidas organizaron la Cumbre Mundial sobre la Sociedad de la Información (CMSI). Uno de los resultados más críticos de esta histórica cumbre fue la creación del Foro de Gobernanza de Internet, o el "IGF". Para más información consultar: https://www.internetsociety.org/es
} 
económicos, así como acceso a los servicios básicos, (...) a las nuevas tecnologías".

- ODS 2 Hambre Cero: Meta 2.a: “Aumentar las inversiones, incluso mediante una mayor cooperación internacional, en infraestructura rural, la investigación agrícola y los servicios de extensión, el desarrollo tecnológico y los bancos de genes de plantas y ganado (...)".

- ODS 4 Educación de Calidad: Meta 4.b: “De aquí a 2020, aumentar considerablemente a nivel mundial el número de becas disponibles para los países en desarrollo (...) a fin de que sus estudiantes puedan matricularse en programas (...) de tecnología de la información y las comunicaciones".

- ODS 5 Igualdad de Género Meta 5.b: “Mejorar el uso de la tecnología instrumental, en particular la tecnología de la información y las comunicaciones, para promover el empoderamiento de las mujeres".

- ODS 8 Trabajo Decente y Crecimiento Económico Meta 8.2: "Lograr niveles más elevados de productividad económica mediante la diversificación, la modernización tecnológica y la innovación (...)", Meta 8.3: "Promover políticas orientadas al desarrollo que apoyen las actividades productivas, la creación de puestos de trabajo decentes, el emprendimiento, la creatividad y la innovación (...)".

- ODS 9 Industria, Innovación e Infraestructura, mencionado previamente.

- ODS 17 Alianzas para Lograr los Objetivos Meta 17.8: "Poner en pleno funcionamiento, a más tardar en 2017, el banco de tecnología y el mecanismo de apoyo a la ciencia, tecnología $e$ innovación para los países menos adelantados y aumentar la utilización de tecnología instrumental, en particular la tecnología de la información y las comunicaciones". 
Como se puede observar en el análisis previo, originariamente, no todos los ODS incluían de manera oficial un componente TIC en la meta correspondiente. Ante esto, la Organización para la Cooperación y el Desarrollo Económicos (OCDE) ha identificado como las TIC podrían contribuir a cada Objetivo concreto incorporando el siguiente análisis a los objetivos restantes ${ }^{8}$ :

- ODS 3 Salud y Bienestar: "El uso de las TIC en el sector de la salud puede dar lugar a una atención sanitaria de mayor calidad que sea más segura y responda en mayor medida a las necesidades de los pacientes. La salud en línea (e-health) cobrará especial importancia en zonas rurales y remotas, al facilitar modelos innovadores de prestaciones sanitarias, como telemedicina $y$ salud móvil'.

- ODS 6 Agua Limpia y Saneamiento - ODS 7 Energía Asequible y No Contaminante: "Las TIC ofrecen la posibilidad de mejorar el acceso al agua y a la energía mediante el uso de soluciones móviles, redes inteligentes o "Smart-grids" y contadores inteligentes que fomentan la eficiencia, gestionan la demanda y desarrollan nuevas formas de ampliar el acceso".

- ODS 10 Reducción de las Desigualdades: "Las TIC, especialmente a través de los servicios móviles, pueden contribuir a reducir la desigualdad al ampliar notablemente el acceso a la información, fomentando así el empoderamiento individual y la inclusión social de personas que solían quedar al margen de los servicios tradicionales".

- ODS 11 Ciudades y Comunidades Sostenibles: "Las TIC se pueden aprovechar para organizar ciudades y comunidades de manera más eficiente. Las ciudades inteligentes utilizan las $T I C y$

\footnotetext{
${ }^{8} \mathrm{La}$ OCDE ha identificado los ejemplos que se describen en los puntos de los ODS 3, 6, $7,10,11,12,13,14,15$ y 16 para ilustrar cómo las TIC podrían contribuir a ese objetivo concreto. Extraído de: OECD/IDB (2016), Políticas de banda ancha para América Latina y el Caribe: Un manual para la economía digital, OECD Publishing, Paris, https://doi.org/10.1787/9789264259027-es.
} 
los datos masivos (Big Data) para mejorar la prestación de servicios públicos y presentar los resultados generales de las políticas, como ahorro energético, la seguridad, la movilidad urbana y el desarrollo sostenible".

- ODS 12 Producción y Consumo Responsables: "Las TIC, y especialmente la banda ancha, han conectado directamente a consumidores y productores dando origen a "mercados a la carta" de productos que se pueden adaptar a requisitos particulares y restringir a una determinada zona, lo que permite ahorrar tiempo, reducir los costos de transporte y fomentar un consumo más eficaz y sostenible".

- $\quad$ ODS 13 Acción por el Clima - ODS 14 Vida Submarina - ODS 15 Vida de Ecosistemas Terrestres: "El uso del Internet de las cosas puede contribuir a que la vigilancia del medio ambiente resulte más barata, rápida y oportuna”.

- ODS 16 Paz, Justicia e Instituciones Sólidas: "El uso de TIC en el sector público puede aumentar la diversidad y el alcance de los servicios de gobierno digital, fortalecer la actuación de las instituciones públicas e incrementar la transparencia y la participación de todos los ciudadanos".

La brecha digital y la falta de capacitación en competencias básicas, como en alfabetización digital, imposibilitan que todas las personas entren en la economía digital y puedan disfrutar de mayores posibilidades laborales. Mediante este trabajo, realizado por la OCDE, se puede ver como Internet contribuye ampliamente al desarrollo sostenible, al progreso de la humanidad. La citada organización también menciona en "Políticas de Banda Ancha para América Latina y El Caribe" que "gracias a la rápida difusión de la banda ancha en todo el mundo, se ha podido reunir evidencia empírica que respalda el efecto clave que esta tecnología digital ha tenido en el crecimiento 
del PIB, la eficacia, los ingresos laborales y el empleo" (OCDE, 2016, p. 14).

La brecha digital resurge en el informe de seguimiento de los Objetivos de Desarrollo Sostenible 2020 que emite la ONU, en el cual se plasma que "en el año 2019, alrededor del $87 \%$ de los hogares de Europa tenían acceso a Internet en sus casas, en comparación con el 18\% de los de África. La brecha digital también se refleja en la propiedad de las computadoras: en 2019, el 78\% de los hogares de Europa tenían una computadora, en comparación con el $11 \%$ de África" (ONU, 2020, p 33).

Tal como surge del análisis previo, la garantía de acceso a Internet no solo es primordial para cerrar la brecha digital, sino para avanzar en la concreción de los Objetivos de Desarrollo Sostenible. El principio de acceso universal "se refiere a la necesidad de garantizar la conectividad y el acceso universal, ubicuo, equitativo, verdaderamente asequible y de calidad adecuada, a la infraestructura de Internet y a los servicios de las TIC, en todo el territorio del Estado" (CIDH, 2013). El ciberespacio es el ámbito que proporciona un cierto carácter de igualdad a todos, igualdad tan ansiada y buscada por la raza humana durante siglos. Por ello, es fundamental cerrar la brecha digital y aquí es donde cumplen un rol fundamental los parlamentarios, tal como se explicará a continuación.

\section{El rol de los parlamentarios en garantizar el acceso a Internet}

Es muy simplista la visión que afirma que el rol del Congreso es "sancionar leyes". Desde ya, es su función esencial. Sin embargo, el rol de los congresos y parlamentos va mucho más allá: son los parlamentarios los que deben garantizar la estabilidad y la legitimidad

\footnotetext{
${ }_{9}$ De esta forma se citan los siguientes estudios: Czernich, 2009; Koutroumpis, 2009; Qiang, Rossotto y Kimura, 2009; BID, 2012a; Thompson y Garbacz, 2008; Bartel, Ichniowski y Shaw 2007; entre otros.
} 
democrática. En este momento, los parlamentos se encuentran frente al enorme desafío de adaptarse a las realidades que presenta el siglo XXI.

Ya se ha desarrollado en extenso, en las secciones anteriores, cómo Internet se presenta como una herramienta fundamental, un derecho en sí mismo que, además, es catalizador de otros derechos. Se ha explicado que promueve el desarrollo sostenible, propicia el crecimiento de la economía digital, también fortalece las capacidades gubernamentales, mientras que su expansión es sustancial para promover la participación ciudadana. Es indudable que permite una gran cooperación entre los gobiernos, y sobre todo frente a una crisis como una pandemia, viabiliza espacios de cooperación virtuales ante el cese de todo tipo de reuniones en persona y viajes internacionales.

El acceso universal a Internet requiere, necesariamente, de la acción de los parlamentarios. Varios fueron los parlamentos que avanzaron en garantizar este derecho emitiendo legislación al respecto, estableciendo que el Estado debe trabajar para asegurar la accesibilidad a Internet, así como restringirse al limitar accesos individuales. Se citan a continuación los de mayor relevancia:

- El Parlamento de Estonia (Riigikogu), el primer país digital del mundo, ejemplo en materia de digitalización y de e-government, fue el primero en sancionar una ley, la Ley de Telecomunicaciones, agregando el acceso a internet entre su lista de servicios universales ${ }^{10}$ (UIT, 2019).

- $\quad$ En 2008, Grecia reformó su constitución e incluyó, entre otras disposiciones, el Artículo 5a y, en particular, su segundo párrafo: "todas las personas tienen derecho a participar de la Sociedad de la Información. La facilitación del acceso a la información transmitida electrónicamente, así como su producción,

\footnotetext{
${ }^{10}$ Artículo 5. 'El conjunto de servicios de telecomunicaciones especificado en el inciso (1) de este apartado comprende: 2) Servicio de Internet disponible universalmente para todos los suscriptores independientemente de su ubicación geográfica, a un precio uniforme".
} 
intercambio y difusión, constituye una obligación del Estado (...)" (Constitución de Grecia, 2008).

- La Unión Europea comenzó, en 2007, un largo proceso legislativo que derivó, dos años después, en 2009, en la Directiva 2009/136/CE, relativa al servicio universal y los derechos de los usuarios en relación con las redes y los servicios de comunicaciones electrónicas (EUR-Lex-2009). Mientras tanto, en 2010, luego de una consulta popular, la UE lanzó el plan de acción sobre la Agenda Digital para Europa, que incluía entre sus objetivos "asegurar que para el 2020 todos los europeos pudieran acceder a una Internet más rápida” (Parlamento Europeo, 2010).

- $\quad$ En 2009, Francia reconoció el derecho fundamental de acceso a Internet, aunque no podemos atribuirle el hecho al Parlamento. En este caso fue la Corte Constitucional francesa quien procedió a semejante declaración, ante una ley del Parlamento contra la piratería ilegal (Daily Mail, 2009).

- En 2010, Finlandia declaró el acceso de banda ancha como un derecho básico, mediante una enmienda a la Ley del Mercado de las Comunicaciones, que establecía que el servicio universal también incluía una conexión funcional a Internet ${ }^{11}$.

- En 2013, México reformó su Constitución Política, incorporando la siguiente norma: "el estado garantizará el derecho de acceso a las tecnologías de la información y comunicación, así como a los servicios de radiodifusión y telecomunicaciones, incluido el de banda ancha e Internet".

- El Congreso argentino sancionó, en el año 2014, la Ley Argentina Digital $\mathrm{N}^{\circ}$ 27.078, que declara de interés público el desarrollo de las TIC y establece como finalidad "garantizar el derecho humano a las comunicaciones y a las telecomunicaciones, 
reconocer a las TIC como un factor preponderante en la independencia tecnológica y productiva de nuestra nación ${ }^{12}$ ".

Los antecedentes descriptos dejan al descubierto el rol, inherente a los parlamentos, de garantizar el derecho fundamental de acceso a Internet. Han pasado 6 años desde la sanción de la Ley $\mathrm{N}^{\circ}$ 27.078, y la Argentina aún no ha concretado el acceso universal a Internet para todos sus habitantes. El Instituto Nacional de Estadística y Censos de la Argentina (INDEC) informa que a fines de 2019 el 79,9\% de la población utilizaba Internet, que el 82,9\% de los hogares tenían acceso a Internet, sin embargo, esto engloba el acceso fijo y móvil y, analizando los números de cerca, solo el 60,9\% de los hogares tenían acceso a una computadora (INDEC, 2019).

La pandemia agravó la situación y las desigualdades sociales. El Observatorio de la Deuda Social Argentina de la Universidad Católica Argentina reafirma, en su informe de marzo 2020, lo que se viene sosteniendo hasta aquí: "los servicios tecnológicos, particularmente los asociados a la conectividad juegan un rol central para la comunicación, al acceso a la información, la cultura y la participación en múltiples redes, este aspecto cobra aún mayor importancia en el contexto de aislamiento" (ODSA, 2020, p.7). Es decir, enfatiza la importancia del acceso a Internet y agrega cifras contundentes: "para el año 2019, según los datos de la Encuesta de la Deuda Social, el 13\% de los hogares no accedía a ningún tipo de conexión a internet, ya sea fija en la vivienda o a través de dispositivos móviles. Esta cifra alcanzaba al 23\% de los hogares de los estratos marginales" (ODSA, 2020, p.7).

En mayo del presente, la organización Asociación Civil por la Igualdad y la Justicia (ACIJ) presentó una demanda contra el Gobierno de la Ciudad de Buenos Aires (GCBA), a fin de que provea Internet en las villas y barrios de emergencia y entregue computadoras a

\footnotetext{
12 Para acceder al texto completo de la norma “Ley Argentina Digital N²7.078" ingresar a: 239999/239771/norma.htm 
préstamo a estudiantes durante la cuarentena. El texto de la demanda ${ }^{13}$ reza: "si bien no hay datos estatales centrados en el acceso a Internet en las villas, la situación de conectividad es sensiblemente menor que las medias nacionales. En este sentido, es necesario tener en cuenta que se estima que el $43 \%$ de la población de las villas porteñas está compuesta por menores de 17 años y el 64,5 por ciento de los hogares tienen niños o adolescentes entre sus integrantes ${ }^{14}$.

Por otro lado, Internet todavía no es considerado un servicio público esencial. En los últimos años, se presentaron más de 10 proyectos en la Cámara de Diputados y en el Senado de la Nación Argentina, con la finalidad de declarar el servicio de telefonía móvil e Internet como servicios públicos esenciales, estratégicos y como un derecho humano.

El proyecto ingresado bajo expediente 2226-D-20, Trámite Parlamentario 49, impulsado por la Diputada María Liliana Schwindt, expone en su artículo 1: "la presente ley tiene como objeto establecer el servicio de telefonía móvil e Internet, en todas sus modalidades y prestaciones como un servicio público esencial, estratégico y un Derecho Humano en los términos y con los alcances de los Artículos 41, 42, 75 inc. 19, 22 y 23 de la Constitución Nacional" (Schwindt, 2020). Varios de esos artículos fueron mencionados previamente, al analizar cómo nuestros constituyentes previeron la inclusión del desarrollo tecnológico dentro de nuestra manda constitucional.

En los fundamentos del proyecto se deja ver como "muchas personas (...) han tenido en la telefonía móvil e internet una posibilidad de acceder a la comunicación, aunque a un costo muy

\footnotetext{
13 Se puede acceder a la Demanda que tramitó ante el Juzgado en lo Contencioso Administrativo y Tributario $\mathrm{N}^{\circ} 2$ de la Ciudad Autónoma de Buenos Aires mediante el siguiente enlace: https://acij.org.ar/acij-demando-al-gcba-para-que-provea-interneten-las-villas-y-entregue-computadoras-en-prestamo-a-estudiantes-durante-lacuarentena/

14 El dato surge de un informe realizado por la Defensoría del Pueblo de la Ciudad Autónoma de Buenos Aires en conjunto con el Observatorio de la Deuda Social de la UCA que abarca a la Villa 31 y 31 Bis, la Villa 20 y el barrio Los Piletones; https://www.tiempoar.com.ar/nota/casi-la-mitad-de-loshabitantes-de-las-villasportenas-son-ninos; 25 de Octubre de 2017
} 
elevado”. Más aún, no solo han accedido a la comunicación, sino a un sinfín de información, a portales educativos y recursos para realizar sus tareas e investigaciones académicas, a diferentes modalidades de trabajo, entre tantos otros usos y recursos.

El proyecto presentado por un grupo de diputados del Frente de Todos (Carro, Osuna y otros, 2020) mediante expediente 2839-D2020, Trámite Parlamentario $\mathrm{N}^{\circ} 65$, reconoce en su artículo 1 que "el acceso a servicios de conectividad e Internet, en sus modalidades de banda ancha fija, móvil y satelital, se considera como derecho humano de carácter universal de conformidad a lo establecido en el art. 2 de la Ley 27.078” y propone la creación de un Plan Nacional de Conectividad que garantice "la máxima cobertura geográfica, la accesibilidad a toda la población, la inclusión digital y la asequibilidad de paquetes de servicios esenciales". Los fundamentos de este proyecto ilustran en detalle la situación actual de la conectividad en Argentina.

En forma similar, otro proyecto presentado por la Senadora Gladis González, también impulsa un plan nacional de conectividad. En los fundamentos, González expresa que:

Es de vital importancia señalar que los ciudadanos puedan acceder al Servicio de Internet, atento a que resulta ser el canal de comunicación de mayor trascendencia en nuestro siglo. Es por este medio que millones de usuarios pueden interactuar entre sí, expresándose de forma libre y, al mismo tiempo, acceden a un vasto intercambio de información sin restricción alguna. (..) además resulta altamente trascendente las múltiples aplicaciones que brinda en materia laboral, científica, industrial, educativa, entre otras (González, 2020).

Existen otros proyectos tendientes al reconocimiento de Internet como Derecho Humano. Entre ellos, se encuentran: el proyecto presentado por el Diputado Pedro Javie Pretto, mediante expediente 1299-D-2019, Trámite Parlamentario 25, que reproduce proyectos del 
mismo autor del año 2017; y el proyecto del Diputado Pablo Carro, mediante expediente 2217-D-2019, Trámite Parlamentario 51. Lamentablemente, estos proyectos han perdido estado parlamentario sin haber sido tratados por el pleno.

La novedad ocurrió en el mes de agosto de 2020 cuando el presidente de la Nación, Alberto Fernández, emitió el Decreto de Necesidad y Urgencia 690/2020, que aplica modificaciones a la Ley $\mathrm{N}^{\circ}$ 27.078 (conocida como "Ley Argentina Digital”) y declara servicio público a los servicios de las TIC, estableciendo, en el artículo 1: “[i]ncorpórase como artículo 15 de la Ley de Tecnologías de la Información y las Comunicaciones, 27.078, el siguiente texto:

Artículo 15: Carácter de servicio público en competencia. Se establece que los servicios de las Tecnologías de la Información y las Comunicaciones (TIC) y el acceso a las redes de telecomunicaciones para y entre licenciatarios $y$ licenciatarias de servicios TIC son servicios públicos esenciales y estratégicos en competencia. La autoridad de aplicación garantizará su efectiva disponibilidad.

El Congreso dio tratamiento al DNU, mediante la discusión en la Comisión Bicameral Permanente de Trámite Legislativo el 24 de agosto de 2020, emitiendo dictamen favorable ${ }^{15}$. Ello es un avance al reconocimiento de Internet como derecho humano dentro de nuestro ordenamiento jurídico, para su garantía de acceso universal. Sin embargo, no es suficiente. Lamentablemente, la labor fundamental del Congreso se ve profundamente desdibujada ante la necesidad de avanzar en el reconocimiento de un derecho en medio de la crisis mundial que ha traído la pandemia.

Como se ha descripto hasta el momento, no se puede negar la trascendencia que tiene el servicio de acceso a Internet. Sin embargo, llegar a garantizarlo como servicio esencial requiere del trabajo

\footnotetext{
${ }^{15}$ Se puede acceder a la versión taquigráfica de la Comisión Bicameral Permanente de Trámite Legislativo mediante el siguiente enlace: https://www.senado.gob.ar/upload/34893.pdf
} 
proactivo de los legisladores. Ellos tienen la obligación de garantizar el derecho a la conectividad en términos estructurales. En este sentido, no solo deben impulsar leyes que promuevan la declaración del acceso a Internet como servicio esencial, sino que, además, deben fomentar la creación de planes estratégicos de conectividad; tarifas sociales para aquellos beneficiarios que no puedan cubrir los costos; regulación de las tarifas por parte del Estado, para que la fijación de precios se de en un marco de razonabilidad; inversión en nuevas tecnologías para asegurar la conectividad constante y un marco especial dentro de la protección de los derechos de los usuarios, para que puedan recibir la prestación del servicio dentro de los niveles de calidad esperables y realizar los reclamos pertinentes en caso de que no se cumplan las condiciones de contratación. A todo ello, se suma un marco en materia de ciberseguridad integral y que vele por la seguridad de todos los ciudadanos y las instituciones en el mundo digital.

\section{El rol de los parlamentos en el ámbito internacional}

Los parlamentos de todos los países deben cooperar para mantener el multilateralismo, para apoyar y contribuir a los aportes realizados por las Naciones Unidas, para fortalecer y promover la cooperación internacional y, aún más, para restaurar el crecimiento económico. En el contexto de la digitalización, es fundamental la cooperación internacional en materia de creación de leyes y regulaciones que permitan a los ciudadanos disfrutar de los beneficios de la tecnología, poniendo como centro los derechos humanos.

No existe un consenso sobre el concepto de "diplomacia parlamentaria”. Tampoco es una materia ampliamente estudiada. En términos simples, la diplomacia parlamentaria es la actividad llevada a cabo por los legisladores en términos de cooperación con parlamentarios de otros países, a fin de "aumentar el control sobre los 
poderes ejecutivos, representar mejor a sus ciudadanos y fortalecer la legitimidad democrática de las instituciones inter-gubernamentales" (Wiesglas y de Boer, 2007).

Esa actividad de cooperación entre los parlamentarios puede darse en forma bilateral o multilateral, a través de instrumentos, como los Grupos Parlamentarios de Amistad, que se forman al interior de cada parlamento. Sin embargo, también existen foros regionales y organismos internacionales, en donde la diplomacia parlamentaria se desarrolla en forma plena. Tal es el caso de la Unión Interparlamentaria (UIP).

La Unión Interparlamentaria es la organización internacional de los parlamentos por excelencia. Como tal, es la única organización que representa a la rama legislativa de los gobiernos a escala mundial, reuniendo a los parlamentos de los Estados soberanos. En la actualidad, la UIP cuenta con 173 parlamentos nacionales miembros ${ }^{16}$.

F. Weisglas y G. de Boer explican que "el mayor involucramiento de los parlamentos en los asuntos internacionales se debe en parte a lo que tan bien se ha denominado "globalización". Cada vez más cuestiones que se ponen a consideración de los parlamentos tienen su origen en desarrollos o estructuras internacionales" (Wiesglas y de Boer, 2007, p. 94). Internet, la banda ancha, el desarrollo de las TIC en general, no son la excepción. Muy por el contrario, pocos temas ocupan la agenda global como las nuevas tecnologías y el progreso de la economía digital a nivel mundial.

Dentro de la Estrategia 2017-2021 de la Unión Interparlamentaria se encuentran diversos objetivos. El "Objetivo 3: Proteger y Promover los Derechos Humanos” insta a los parlamentos a "aplicar normas internacionales de derechos humanos (...) abordando las cuestiones actuales y más acuciantes relacionadas con

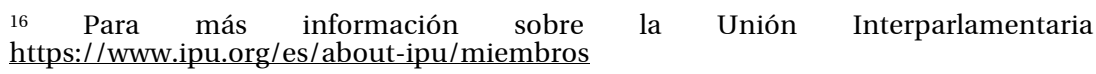


estos". Sumado a ello, el "Objetivo 7: Movilizar a los parlamentos en favor de la agenda mundial del desarrollo" se enfoca en aumentar las capacidades de los parlamentos para que estos puedan poner en práctica los ODS, supervisar su avance y fomentar su cumplimiento (UIP, 2017). Ya hemos visto previamente como todos los ODS poseen expresa o implícitamente un componente TIC que alienta su pleno cumplimiento dentro del contexto digital.

La diplomacia parlamentaria, ya sea la que se da en foros y organismos interparlamentarios o de forma bilateral o multilateral, no solo permite la cooperación entre parlamentos para fortalecer las instituciones democráticas y compartir mejores prácticas. Se presenta, por sobre todo, como una herramienta fundamental para permitir a los países examinar problemas concretos o urgentes, así como diferentes formas de abordarlos.

El último informe de la Unión Interparlamentaria, en materia de e-parliament, muestra algunos puntos clave para tener en cuenta, no solo a fin de que los parlamentarios avancen en el acceso universal a Internet, sino en la propia incorporación de las TIC al interior de las instituciones. Entre ellos se destaca el hecho de que "algunos obstáculos para la utilización mayor de las TIC son la falta de conocimientos y de capacitación de los parlamentarios y del personal y una preocupación creciente sobre su seguridad y fiabilidad" (UIP, 2018, p.6). Avanzar en la garantía de acceso a Internet por parte de la población de cada país, también redundaría en la incorporación de herramientas digitales en el ámbito gubernamental, entre ellos los organismos parlamentarios. Internet es una herramienta fundamental para garantizar el acceso a la información, fortaleciendo la rendición de cuentas, y promover la participación ciudadana que viabiliza el involucramiento de la ciudadanía con el parlamento. Además, la incorporación de las TIC mejora los servicios parlamentarios y aumenta la transparencia.

Sin lugar a dudas, debe existir mayor capacitación y colaboración con expertos para poder lograr avances. La UIP afirma que: 
Existe una tradición de larga data basada en colaborar, compartir e intercambiar entre parlamentos (...) las redes interparlamentarias internacionales siguen siendo populares. La pertenencia a la Alianza para el Gobierno Abierto (OGP, Open Government Partnership) ha crecido, mientras que la Federación Internacional de Asociaciones de Bibliotecarios y Bibliotecas (IFLA, International Federation of Library Associations and Institutions) permanece como la red preferida por las bibliotecas parlamentarias y conforma la agrupación interparlamentaria de mayor tamaño (UIP, 2018, p.28).

La gobernanza de Internet debería ocupar un lugar central en la agenda política, en cuyo debate deben participar como figuras centrales los parlamentos, junto a los poderes ejecutivos, pero también el sector privado, la academia y la sociedad civil, así como los usuarios, todos en igualdad de condiciones. La gobernanza de Internet no solo implica su regulación, sino garantizar su acceso universal, su funcionamiento estable y seguro, contemplar las diferencias culturales para incluir las múltiples lenguas, entre otras (Aspis, 2014). A fin de obtener una gobernanza de Internet eficaz y completa, es necesaria la cooperación intergubernamental y, en esto, la diplomacia parlamentaria juega un rol primordial.

En los últimos encuentros del Parliamentary 20 (P20), la Cumbre de Presidentes y Presidentas de Parlamentos en el ámbito del G20, los participantes abordaron los diversos temas relacionados con la gobernanza global, a fin de generar conciencia y apoyo político para alcanzar los compromisos internacionales que aseguren que estos se traduzcan en realidades locales.

La última edición del P20 se realizó en Osaka, en noviembre de 2019. En la segunda sesión, denominada "la utilización de tecnología innovadora para lograr una sociedad futura centrada en el ser humano", se presentó un debate sobre los méritos de utilizar la inteligencia artificial, el Internet de las cosas y otras tecnologías, para conseguir una sociedad que se centre en las personas (UIP, 2019). Al 
finalizar la jornada, como es costumbre, se adoptó por unanimidad una declaración conjunta. Entre sus principales definiciones se instó al G20 "a aprovechar la Inteligencia Artificial (IA) y el Internet de las cosas para lograr una sociedad centrada en el ser humano, en la que mejore la productividad y el crecimiento inclusivo" y a "promover el flujo seguro y ordenado de información y a permitir a los usuarios de Internet acceder legalmente a la información, el conocimiento y los servicios en línea", reafirmándose "el apoyo parlamentario al combate contra el extremismo, la xenofobia y el terrorismo en todas sus formas y manifestaciones ${ }^{17}$ ". Nada de ello será posible sin la garantía universal del acceso a Internet y el cierre de la brecha digital. Las reuniones de trabajo en el ámbito de la UIP, el P20 y otras reuniones multilaterales y bilaterales entre miembros de diferentes parlamentos, deberían traducirse en acciones concretas con impacto real en promover el acceso a Internet.

\section{Conclusión}

En los últimos 10 años —y de forma precipitada — Internet pasó de ser una mera herramienta tecnológica que viabilizaba el acceso a la información y las comunicaciones a transformarse en un derecho fundamental por sí mismo, catalizador de otros derechos. El acceso a las TIC tiene la capacidad de mejorar la calidad de vida de todos los pueblos, sobre todo de las comunidades más vulnerables.

No solo los ciudadanos se benefician del avance tecnológico, sino que las instituciones avanzan en procesos más ágiles y servicios de mayor calidad. A través de Internet, los ciudadanos tienen la oportunidad única de participar en forma activa con los gobiernos, acceder a la información y reforzar la rendición de cuentas. Internet es un elemento democratizador, promotor del derecho a la

17 La declaración conjunta puede consultarse en inglés en el siguiente enlace https://www.ipu.org/news/press-releases/2019-11/p20-strengthens-parliamentarydimension-g20 
educación, a la cultura, a la comunicación, a nuevas formas de trabajo y al derecho a la salud.

La ONU se destaca como la institución que más esfuerzos realizó al resaltar la importancia de garantizar el acceso universal y asequible a Internet. Como se ha descripto en el desarrollo del presente trabajo, Internet y las TIC tienen la capacidad de favorecer la concreción de los 17 Objetivos de Desarrollo Sostenible de la Agenda 2030 de Naciones Unidas.

Para ello, los parlamentarios no pueden desconocer su rol central al ser los promotores de leyes que reconozcan que Internet es un derecho humano, estableciéndolo como servicio esencial y garantizando su acceso universal. Se ha descripto cómo avanzaron varios países del mundo. En Argentina, se presentaron diversos proyectos de ley ante ambas Cámaras del Congreso, pero ninguno llegó a tener tratamiento. Sin la proactividad necesaria no podremos cerrar la brecha digital, que se hace más visible en tiempos de crisis sanitaria y aislamientos prolongados. El reconocimiento del acceso a Internet como derecho humano no debería tener que inferirse de la letra poco clara de la legislación comentada, como la ley Argentina Digital. Dada la importancia cardinal de este derecho, su reconocimiento, garantía y cumplimiento en la práctica deberían ser mucho más enfáticos y precisos.

La diplomacia parlamentaria, como herramienta fundamental al servicio de los legisladores, se presenta como una oportunidad única para fortalecer la cooperación, intercambiar buenas prácticas y asumir compromisos conjuntos que permitan garantizar el acceso universal a Internet. La UIP, organismo internacional que reúne la mayor cantidad de parlamentos del mundo, no solo brinda un espacio para que los parlamentarios fortalezcan las instituciones, sino que abre paso a discusiones sobre materias específicas que afectan a todos los habitantes del mundo. Internet, es sin dudas, una de las materias más apremiantes para que la globalización y el desarrollo sostenible sean una realidad compartida por todos, en igualdad de condiciones. 


\section{Bibliografía}

Águila, I. J. (2012). Internet, un derecho humano de cuarta generación. Universidad Colegio Mayor de Cundinamarca. Recuperado de: https://revistas.unicolmayor.edu.co/index.php/mjuridica/articl e/view/369/688

Asociación Civil por la Igualdad y la Justicia (ACIJ). (2020). Demandamos al GCBA para que provea Internet en las villas y entregue computadoras en préstamo a estudiantes durante la cuarentena. Recuperado de: https://acij.org.ar/acij-demandoal-gcba-para-que-provea-internet-en-las-villas-y-entreguecomputadoras-en-prestamo-a-estudiantes-durante-lacuarentena/

Aspis, A (2014) La gobernanza de Internet y la nueva agenda mundial de los recursos tecnológicos. $14^{\circ}$ Simposio Argentino de Informática y Derecho, SID 2014. ISSN: 1850-2814

BBC. (2010). Four in Five regard Internet access as fundamental right: global poll. Press Office, World Service. Recuperado de: http://www.bbc.co.uk/pressoffice/pressreleases/stories/2010/0 3_march $/ 07 /$ poll.shtml

Bidegain, C. M. (1994-2001). Curso de Derecho Constitucional. Buenos Aires, Argentina: (Nva. ed. rev. y act. con la reforma de 1994 ed.). Abeledo-Perrot S.A.

Carro, P. (2019). Proyecto de Ley: Reconocimiento del acceso al servicio de Internet como Derecho Humano. Expediente Expediente 2217-D-2019, Trámite Parlamentario 51. Honorable Cámara de Diputados de la Nación. Recuperado de: https://www.diputados.gov.ar/proyectos/proyectoTP.jsp?exp=2 217-D-2019

Carro, P.; Osuna, B. y otros. (2020). Proyecto de Ley: Acceso a Internet como Derecho Humano y Servicio Universal. Plan Nacional de Conectividad con Prioridad Educativa. Expediente 2849-D-2020, Trámite Parlamentario N65. Honorable Cámara de Diputados de la Nación. Recuperado de: https://www4.hcdn.gob.ar/dependencias/dsecretaria/Periodo2 020/PDF2020/TP2020/2849-D-2020.pdf 
Constitución de la Nación Argentina: http://servicios.infoleg.gob.ar/infolegInternet/anexos/04999/804/norma.htm

Corte Interamericana de Derechos Humanos (CIDH). (2013). Informe de la Relatoría Especial para la Libertad de Expresión. Capítulo IV (Libertad de Expresión e Internet). Recuperado de:http://www.oas.org/es/cidh/expresion/docs/informes/anua les/2014_04_22_IA_2013_ESP_FINAL_WEB.pdf

Corte Interamericana de Derechos Humanos (CIDH). (2016). Estándares para una Internet libre, abierta $e$ incluyente. Recuperado de: http://www.oas.org/es/cidh/expresion/docs/publicaciones/IN TERNET_2016_ESP.pdf

Daily Mail. (2009). Internet access is a fundamental human right, rules French court. Recuperado de: https://www.dailymail.co.uk/news/article-1192359/Internetaccess-fundamental-human-right-rules-Frenchcourt.html\#: :text=Access\%20to\%20the\%20internet\%20is,claim \%20France's\%20most\%20senior\%20lawmakers.\&text='The\%20i nternet\%20is\%20a\%20fundamental,'

EUR-Lex. (2009). Directiva 2009/136/CE. Recuperado de: https:/ / eurlex.europa.eu/legal-content/ES/TXT/?uri=celex\%3A32009L0136

Gobierno de México. (2013). En México, el acceso a internet es un derecho constitucional. Recuperado de: https://www.gob.mx/gobmx/articulos/en-mexico-el-acceso-ainternet-es-un-derecho-

constitucional\#: :text=A\%20partir\%20de\%20la\%20Reforma,tod as\%20las\%20mexicanas\%20y\%20mexicanos.\&text=En\%20M\%C 3\%A9xico\%2C\%20el\%20acceso\%20a\%20internet\%20es\%20un\% 20derecho\%20garantizado,Constituci\%C3\%B3n\%20Pol\%C3\%A Dtica\%20de\%20nuestro\%20pa\%C3\%ADs.

Gonzalez, G. (2020). Proyecto de ley que declara como derecho humano el servicio público a la telefonía móvil e internet. Expediente 1100-S-2020 Diario de Asuntos Entrados 52. Honorable Senado de la Nación. Recuperado de: https://www.senado.gob.ar/parlamentario/comisiones/verExp /1100.20/S/PL 
Instituto Nacional de Estadística y Censos INDEC (2019). Tecnología, Informes Técnicos. Recuperado de: https://www.indec.gob.ar/indec/web/Nivel3-Tema-4-26

La Constitución de Grecia (The Constitution of Greece) (2008) Recuperada http://www.hellenicparliament.gr/UserFiles/f3c70a23-769649db-9148-f24dce6a27c8/001-156\%20aggliko.pdf

Ley Argentina Digital $\mathrm{N}^{\circ}$ 27.078, Recuperada de: http://servicios.infoleg.gob.ar/infolegInternet/anexos/235000239999/239771/norma.htm

Ley del Mercados de las Comunicaciones de Finlandia (Finland Communications Market Act) Recuperada de: https://www.finlex.fi/en/laki/kaannokset/2003/en20030393.pdf

Macklem, P. (2015). Human rights in international law: three generations or one? London Review of International Law, 3(1), 61-92. doi:10.1093/lril/lrv001

Naciones Unidas [ONU]. (2020). Informe de los Objetivos de Desarrollo Sostenible 2020. Recuperado de: https://unstats.un.org/sdgs/report/2020/The-SustainableDevelopment-Goals-Report-2020_Spanish.pdf

Naciones Unidas. (2011). Informe del Relator Especial sobre la promoción y protección del derecho a la libertad de opinión y de expresión, Frank La Rue (A/HRC/17/27) Recuperado de: https://www.acnur.org/fileadmin/Documentos/BDL/2015/100 48.pdf

Naciones Unidas. (2016). Promoción, protección y disfrute de los derechos humanos en Internet (A/HRC/32/L.20) Recuperado de: https://ap.ohchr.org/documents/S/HRC/d_res_dec/A_HRC_32 _L20.pdf

Observatorio de la Deuda Social Argentina (ODSA). (2020). Desigualdades Sociales en Tiempos de Pandemia. Universidad Católica Argentina. Recuperado de: http://wadmin.uca.edu.ar/public/ckeditor/Observatorio\%20De uda\%20Social/Documentos/2020/OBSERVATORIOCOMUNICADO-ODSA\%20INFORMA-\%202-31_03_VF.pdf 
OCDE. (2016). Políticas de banda ancha para América Latina y el Caribe: Un manual para la economía digital. OECD Publishing, Paris. Recuperado de: http://www.oecd.org/internet/broadband/lac-digitaltoolkit/Home/LAC-Broadband-Toolkit-ESP-Excerpt.pdf

ONU News. (2017). Las tecnologías de la información son fundamentales para responder a los desafíos del desarrollo. Recuperado de: https://news.un.org/es/story/2017/07/1382531

Organización de los Estados Americanos (OEA). (2011). Relatorías de libertad de expresión emiten declaración conjunta acerca de Internet. Comunicado de Prensa R50/11. Recuperado de: https://www.oas.org/es/cidh/expresion/showarticle.asp?artID= 848

Parlamento Europeo. (2010). Fichas temáticas sobre la Unión Europea: Una agenda digital para Europa. Recuperado de: https://www.europarl.europa.eu/factsheets/es/sheet/64/unaagenda-digital-para-europa

Pretto, P. (2017). Proyecto de Ley: Reconocimiento del Acceso al Servicio de Internet como Derecho Humano. Expediente 6380D-2017, Trámite Parlamentario 177. Honorable Cámara de Diputados de la Nación. Recuperado de: https://www.hcdn.gob.ar/proyectos/proyectoTP.jsp?exp=6380D-2017

Reglitz, M. (2019). The Human Right to Free Internet Access. Journal of Applied Philosophy. https://doi.org/10.1111/japp.12395

Rothkopf, D. (2015). Rights 2.0 Is Unrestricted Internet Access a Modern Human Right? Foreign Policy $N^{\circ} 10$. Recuperado de https://www.questia.com/magazine/1G1-399884300/rights-20-is-unrestricted-internet-access-a-modern

Schwindt, M. (2020). Proyecto de Ley: Establecer Al Servicio De Telefonía Móvil E Internet Como Servicio Público Esencial, Estratégico $Y$ Derecho Humano. Expediente 2226-D-2020, Trámite Parlamentario $\mathrm{N}^{\circ} 49$. Honorable Cámara de Diputados de la Nación. Recuperado de: https://www4.hcdn.gob.ar/dependencias/dsecretaria/Periodo2 020/PDF2020/TP2020/2226-D-2020.pdf

Unión Internacional de Telecomunicaciones. (2019). New ITU data reveal growing Internet uptake but a widening digital gender 
divide

Recuperado

de:

https://www.itu.int/en/mediacentre/Pages/2019-PR19.aspx

Unión Interparlamentaria (UIP). (2017). Estrategia 2017-2021. Recuperado http://archive.ipu.org/pdf/publications/strategy1721-s.pdf

de:

Unión Interparlamentaria (UIP). (2018). World e-Parliament Report 2018. Recuperado

de:

https://www.ipu.org/resources/publications/reports/201811/world-e-parliament-report-2018

Unión Interparlamentaria (UIP). (2019). P20 strengthens parliamentary dimension of G20. Recuperado de: https://www.ipu.org/news/press-releases/2019-11/p20strengthens-parliamentary-dimension-g20

Vasak, Karel (1977). Human Rights: A Thirty-Year Struggle: the Sustained Efforts to give Force of law to the Universal Declaration of Human Rights. UNESCO Courier, 11:29-32.

We Are Social. (2020). Special Reports: Digital 2020. 3.8 billion people use social media. Recuperado de: https://wearesocial.com/blog/2020/01/digital-2020-3-8-billionpeople-use-social-media

Weisglas, F.; de Boer, G (2007). Parliamentary Diplomacy, The Hague Journal of Diplomacy 2, pp. 93-99, The Netherlands. 